\title{
Hardware Approach of R-Peak Detection for the Measurement of Fetal and Maternal Heart Rates
}

\author{
M. A. $\operatorname{Hasan}^{1}$, Md Mamun² \\ ${ }^{1}$ School of Electrical and Electronic Engineering \\ The University of Adelaide \\ South Australia 5005, Australia \\ ${ }^{2}$ Department of Electrical, Electronic and Systems Engineering \\ Universiti Kebangsaan Malaysia \\ 43600 UKM, Bangi, Selangor, Malaysia \\ *mdmamun.ukm@gmail.com
}

\begin{abstract}
Fetal heart rate (FHR) monitoring is a routine for obtaining significant information about the fetal condition during pregnancy and labour. Fetal condition may change abruptly during the pregnancy period. Therefore, a continuous fetal electrocardiogram (FECG) monitoring will ease the fetal well-being. An algorithm has been developed to detect R-peak for the simultaneous measurement of the fetal and maternal hearts rates during pregnancy and labor for fetal monitoring. The algorithm is based on a cross-correlation, adaptive threshold and statistical properties in the time domain. The performance achieved for the R-peak detection for the heart rate measurements shows that the model can extract R-peak for both, mother and fetus utilizing a single-lead configuration. The algorithm has been implemented in the field of propagation gate array (FPGA). The design was synthesized and fitted into Altera's Stratix EP1S10 using the Quartus II platform because of its enhanced DSP capability. Test case results showed an error percentage of around $\pm 0.3 \%$ and $\pm 0.5 \%$ for the R-peak detection of maternal and fetal mortality respectively. The system is capable of running at a maximum clock frequency of $48.56 \mathrm{MHz}$, and consumes 9633 logic elements, 101616 memory space and 4 units of DSP blocks.
\end{abstract}

Keywords: FPGA, Electrocardiogram, VHDL, DSP, Fetal heart rate, QRS.

\section{RESUMEN}

EL monitoreo de la frecuencia cardiaca fetal (FHR) es una rutina para obtener información significativa sobre el estado del feto durante el embarazo y el parto. La condición fetal puede cambiar abruptamente durante el período de embarazo. Por lo tanto, un continuo monitoreo fetal por medio de electrocardiograma (FECG) facilitará el bienestar fetal. Se ha desarrollado un algoritmo para detectar el pico R para la medición simultánea de las frecuencias cardiacas fetal y materna durante el embarazo y el trabajo de monitorización fetal. El algoritmo se basa en la correlación cruzada del umbral adaptativo y propiedades estadísticas en el dominio del tiempo. El desempeño obtenido para la detección del pico $\mathrm{R}$ para las mediciones del ritmo cardíaco muestra que el modelo puede extraer picos $\mathrm{R}$ para ambos madre y feto utilizando una configuración de una sola via. El algoritmo ha sido implementado en el arreglo de compuertas de propagación (FPGA). El diseño fuésintetizado y acomodado en Stratix de Altera EP1S10 utilizando la plataforma Quartus II debido a su capacidad DSP mejorada. Los resultados de las pruebas de caso mostraron un porcentaje de error de alrededor de $\pm 0,3 \%$ y $\pm 0,5 \%$ para la detección R-pico de la mortalidad materna y fetal, respectivamente. El sistema es capaz de funcionar a una frecuencia de reloj máxima de $48.56 \mathrm{MHz}$, y consumes 9633 elementos lógicos, 101616 espacio de memoria y 4 unidades de bloques DSP.

\section{Introduction}

The electrocardiogram (ECG) is the electrical signal produced by the heart and contains the distinctive shape known as the QRS complex. The time between two successive $R$ peaks of the QRS complex is known as the RR interval and the heart rate (HR) is the reciprocal of the RR interval expressed in Beat Per Minute (BPM). Electronic fetal heart rate (FHR) monitoring is used to determine if the fetus is free from any complications such as antenatal uteroplacental insufficiency and fetal hypoxia, and to determine the fetal health [1]. Continuous efforts are being made to produce more efficient and accurate methods to extract the fetal RR interval, and gain a better interpretation of the FHR patterns by researchers. 
At present, Doppler ultrasound has become a popular technique of monitoring the FHR abdominally but attempts to produce a portable system have not been successful because of its sensitivity to movements [2]. A method utilizing the abdominal electrocardiogram (AECG) has a better prospect for long-term monitoring but requires much signal processing to be done $[2,3]$. This method is non-invasive and has potential to convey the electro- physiological information, which helps to determine the conditions of the fetus such as stress and acidosis, and uterine activity $[3,4]$. The disadvantages of such systems require intermittent repositioning of the transducer and they are only suitable for use with highly trained midwifes. The ultrasound transducer is problematic and uncomfortable while the procedure involves launching a $2 \mathrm{MHz}$ signal towards the fetus. The use of Doppler ultrasound (invasive manner) is not suitable for long periods of FHR monitoring [5-7]. Doppler ultrasound technique suffers from the limitation of sensitivity to movement and most Doppler systems rely upon some form of averaging to produce their FHR data. In contrast, methods utilizing the AECG have a greater prospect for long-term monitoring of FHR and fetal well-being using signal processing techniques. The AECG signal can also be used for antepartum non-invasive FHR determination through the detection of small fetal cardiac potentials at the surface of the maternal abdomen [8]. The AECG can be used to produce true RR interval data, which is suitable for heart rate variability studies if required. Its advantages are that it is completely non-invasive and unobtrusive, has comparatively low power requirements and can be used over extended (e.g. 24 hours) periods. The method additionally allows the maternal heart rate (MHR) to be recorded since the MECG is also detected from the AECG. The advantage of using AECG to extract FECG is that additional information can be extracted compare to using Doppler ultrasound although the accuracy of this technique is much lower compare to the former one [9].

The Field-programmable gate arrays (FPGA) offers a potential alternative to speed up the hardware realization $[10,11]$. From the perspective of computer-aided design, FPGA comes with the merits of lower cost, higher density, and shorter design cycle [12]. It comprises a wide variety of building blocks. Each block consists of programmable look-up table and storage registers, where interconnections among these blocks are programmed through the hardware description language [13]. This programmability and simplicity of FPGA made it favorable for prototyping digital system. FPGA allows the users to easily and inexpensively realize their own logic networks in hardware. FPGA also allows modifying the algorithm easily and the design time frame for the hardware becomes shorter by using FPGA [14].

In this work we propose the framework of FPGAbased hardware realization of fetal heart-rate detection algorithm. The VHDL is selected as the hardware description language, which could be used to realize the maternal and fetal QRS complex extraction to measure both the FHR and maternal heart rate (MHR). The use of VHDL for modeling is especially appealing since it provides a formal description of the system and allows the use of specific description styles to cover the different abstraction levels (architectural, register transfer and logic level) employed in the design [11]. In the method computation of, the problem is first divided into small pieces; each can be seen as a submodule in VHDL. Following the software verification of each submodule, the synthesis is then activated. It performs the translations of hardware description language code into an equivalent netlist of digital cells. The synthesis helps integrate the design work and provides a higher feasibility to explore a far wider range of architectural alternative [12]. In this study, to validate the effectiveness of the method, various maternal ECG have been used. The method provides a systematic approach for hardware realization, facilitating the rapid prototyping of Fetal heart-rate detection.

\section{Clinical significance of FECG morphology}

Biomedical signal implies a collective electrical signal acquired from any organ that represents a physical variable of interest where the signal is

considered in general a function of time and is describable in terms of its amplitude, frequency, and phase. FECG is a biomedical signal that provides electrical representation of FHR to get the vital information about the condition of fetus during gestation and labor from the recordings on the 
mother's body surface. Sometimes the FECG is the only information source in early stage diagnostic of fetal health and status. The FECG signal is very much related to the adult ECG, containing the same basic waveforms including the P-wave, the QRS complex, and the T-wave. The PQRST complex as shown in figure 1 is an electric signal produced by the contraction of the heart's muscle called myocardium. It is composed of three parts; first, the P-wave reflects the contraction of the atriales. Second, the QRS-complex is related to the contraction of the ventricles, due to the magnitude of the R-wave, it is extremely reliable. Finally, the T-wave, which corresponds to the repolarization phase, which follows each heart contraction.

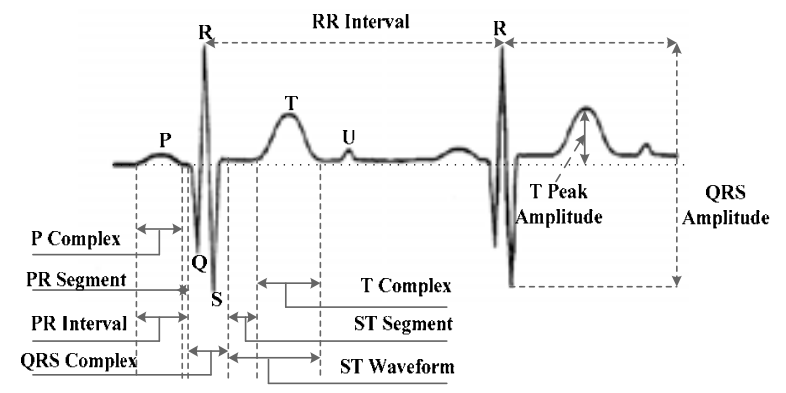

Figure1. FECG shows key features: the PQRST complex

\section{Methodology}

\subsection{R-peak detection of maternal QRS}

The detection of maternal QRS complexes begins with cross-correlating the signal with an average maternal QRS template. The cross-correlation output of the signal $\mathrm{x}$ at each instant $\mathrm{n}$ with the template $\mathrm{s}(\mathrm{k})$ is given by

$$
y(n)=\sum_{k=0}^{M} h(k) x(n-k)
$$

where, $h(k)= \begin{cases}s(M-k), & 0 \leq k \leq M \\ 0, & \text { elsewhere }\end{cases}$

The template $s(k)$ with $(M+1)$ equally spaced points over $80 \mathrm{~ms}$ has been empirically found to be optimized for the detection of maternal QRS complexes when $M=8$. The width of the template is based on the normal width of the maternal QRS complex [15]. The template is continuously updated with the detection of $R$ peaks to take into consideration the variation of shape of the maternal QRS complexes in AECG.

The local maxima search routine measures the slope of the cross-correlated output by

$$
y^{\prime}(n)=y(n)-y(n-1)
$$

It also assumes a maximum at sample $(n-1)$ when the slope changes from $y^{\prime}(n-1) \geq 0$ to $y^{\prime}(n)<0$. If no maximum is found in the subsequent $20 \mathrm{~ms}$ (assumed to be the minimum fetal QRS duration), the sample value $y(n-1)$ and corresponding instant are saved as the local maximum. This $20 \mathrm{~ms}$ search interval is necessary to avoid taking small spikes on the slopes of the QRS complexes as maxima.

Three values, $V_{\mathrm{M} 1}>\mathrm{V}_{\mathrm{M} 2}>\mathrm{V}_{\mathrm{M} 3}$, and their time instants corresponding to the largest three local maxima are stored within an $\mathrm{R}$ wave search interval. The length of the search interval is initially one second (in fact $1024 \mathrm{~ms}$ for computational simplicity) and it is then continuously updated after the first RR interval measurement. The one-second search interval and the saving of 3 local maxima assume that the maternal heart rate (MHR) does not exceed 120 BPM which means at most 2 maternal $R$ peaks can be found in the initial search interval. If $\mathrm{V}_{\mathrm{M} 1}$ is validated as the $\mathrm{R}$ peak then the value $V_{M 2}$ is taken as the noise. $V_{M 3}$ is kept for cases when $V_{M 2}$ is validated as the $R$ peak. The threshold used in the detection is set initially by assuming a minimum maternal $\mathrm{R}$ peak of $10 \mu \mathrm{V}$ and it is continuously updated based on the levels of both $R$ peak and noise [16]. A possible maternal $R$ peak is assumed to be found when the value $V_{M 1}$ exceeds this threshold. $V_{\mathrm{M}_{2}}$ is also considered as an $R$ peak if the value is comparable to that of $V_{M 1}$ and the resulting heart rate is below $120 \mathrm{BPM}$, as earlier assumed. Hence the criteria:

$$
2 V_{M 2}>V_{M 1}
$$

and

$$
\left|t_{M 2}-t_{M 1}\right|>512 m s
$$


If $V_{\mathrm{M} 2}$ also exceeds the threshold, the QRS template is compared with the complexes associated with both $\mathrm{V}_{\mathrm{M} 1}$ and $\mathrm{V}_{\mathrm{M} 2}$. The one with the least mean square error is taken to be the $\mathrm{R}$ peak. The other peak is assumed to be a large spike in the signal and its position is saved for use in the fetal $R$ peak validation routine. If $V_{M 2}$ has the larger error, its position is saved only if inequality in (3) applies, because smaller $\mathrm{V}_{\mathrm{M} 2}$ may be associated with an actual fetal $\mathrm{R}$ peak.

The running average used in this algorithm is performed to average the QRS templates, RR intervals, levels of $R$ peak and noise. The $b$-th value of the running average $A(b)$ is given by $a$ weighting of the previous average $A(b-1)$ plus that of the new value $C(b)$ as shown in the following equation:

$$
A(b)=\{1-k(b)\} A(b-1)+k(b) C(b)
$$

where, $k(b)= \begin{cases}\frac{1}{b}, & b \leq B \\ \frac{1}{B}, & b>B\end{cases}$

The running averages of noise and $R$ peaks $\left(A_{N}\right.$ and $A_{R}$ ) are estimated over $B$ recent values where, $B=8$ in (5) has been empirically found to be effective. Based on these averages, two thresholds, $\mathrm{TM}_{1}$ and $\mathrm{TM}_{2}$ are used in the $\mathrm{R}$ wave search. They are given by

$$
\begin{aligned}
& T M_{1}=A_{N}+\frac{A_{R}-A_{N}}{4} \\
& T M_{2}=\frac{T M_{1}}{2}
\end{aligned}
$$

The adaptation of the threshold to varying $R$ peak and noise levels, and the $R$ wave search interval are based on the method proposed in [17].

If the maximum search limit is reached while the local maximum $V_{M 1}$ has a value less than $T_{1}$, then $V_{M 1}$ is taken as a possible $R$ peak if it exceeds the second threshold, $\mathrm{TM}_{2}$. If no such $\mathrm{V}_{\mathrm{M} 1}$ is found, a signal loss is assumed. The local maxima values are then set to zero for the subsequent $R$ wave search. Four latest maternal RR intervals are maintained in record for the purpose of checking coincidences of the maternal with the fetal $R$ waves.

\subsection{R-peak detection of fetal QRS}

The maternal electrocardiogram (MECG) complex is then subtracted upon detection of a maternal QRS to remove the maternal contribution from the abdominal signal. This complex is of fixed duration, $160 \mathrm{~ms}$ before and $320 \mathrm{~ms}$ after the maternal $\mathrm{R}$ peak instant. This duration assumes that the average MHR is less than 125 BPM and it should normally include the $P$ and $T$ waves, if any. The MECG template is matched with actual MECG in the abdominal signal by scaling it with the factor,

$$
K=\sqrt{\frac{\text { Value1 }}{\text { Value2 }}}
$$

where, Value1 < Value2. These values are obtained from the cross-correlation of abdominal signal with maternal template and auto correlation of the maternal template. If the cross correlation value is greater than the auto correlation value, then the abdominal signal is multiplied by the factor $\mathrm{K}$ and MECG template is subtracted, if not, MECG template is multiplied by factor $\mathrm{K}$ and subtracted from the abdominal ECG signal.

The detection of the fetal QRS complex begins by differencing local maxima and minima on the output of the subtracted signal when the time marker counter, which was initiated at the second accepted maternal R peak, has reached 2048 ms [18]. This duration ensures that the 2 second delayed samples are already within the MECG subtracted region of the signal. Observing the waveforms, it is possible to differentiate between fetal events and noise events if the amplitudes are similar. This is partly because of the rapid and large deflections between a local maximum and the following local minimum when a fetal beat has occurred. From (2), a minimum is assumed at sample $(n-1)$ when the slope changes from $y^{\prime}(n-1)$ $<0$ to $y^{\prime}(n) \geq 0$. The absolute value of the difference between successive peak and valley is computed for each max-to-min interval.

The local maxima search routine is performed on the output of the differencing of local maxima and minima routine, and three largest maxima, $V_{F 1}>$ 
$\mathrm{V}_{\mathrm{F} 2}>\mathrm{V}_{\mathrm{F} 3}$ are kept as before. The initial search interval is $640 \mathrm{~ms}$ so that at most two fetal $R$ peaks can be found by assuming the FHR does not exceed 187 BPM during the initial search interval. The first search is repeated for another subsequent $640 \mathrm{~ms}$ if the largest local maximum, $V_{F 1}$ is concurrent with a maternal QRS complex and $V_{F 2}$ is smaller than a threshold or is also concurrent. The threshold used in the FHR detection is set initially by assuming a minimum fetal $\mathrm{R}$ peak of $5 \mu \mathrm{V}$ and it is continuously updated [17]. The routine is similar to that for the maternal case but uses the following criteria to accept $V_{F 2}$ as a possible fetal $\mathrm{R}$ peak:

$$
1.5 V_{F 2}>V_{F 1}
$$

and

$$
\left|t_{F 2}-t_{F 1}\right|>320 m s
$$

The second search is repeated if the accepted first fetal $R$ peak is found to be concurrent with a maternal QRS complex or if

$$
2 V_{F 3}>V_{F 1}
$$

i.e. the signal is noisy with all its three local maxima having comparable values. The fetal and maternal QRS complexes are concurrent if

$$
\left|t_{F}-t_{M}\right|<64 m s
$$

where $t_{F}$ and $t_{M}$ are the fetal and maternal $R$ peak instants, respectively. The range in (12) accounts for possible overlap of the two complexes, which are assumed to have widths of 50 and $80 \mathrm{~ms}$ respectively. The overlap is checked by relating the fetal $R$ peak instant to the four latest maternal RR intervals.

The subsequent fetal $\mathrm{R}$ wave detection procedure is the same as that for the maternal $\mathrm{R}$ wave using two thresholds, $\mathrm{TF}_{1}$ and $\mathrm{TF}_{2}$ which are set as in (6) and (7), according to the running average of the $R$ peaks and noise with $B=8$ in (5). The determination of the fetal $R$ wave search interval is also based on the method proposed in [18]. The second threshold, $\mathrm{TF}_{2}$ is used when the maximum search limit is reached. A signal loss is assumed when no maximum exceeding the threshold is found. When the second threshold is used to identify a fetal $\mathrm{R}$ peak, the peaks are averaged with $B=4$ so that the first threshold will quickly adapt to the smaller signal.

After a possible fetal $R$ wave is found, a continuation of the search for up to $220 \mathrm{~ms}$ is carried out unless the maximum search limit is reached. This forward searching reduces the possibility of false $R$ wave detection with the assumption that the heart rate does not exceed 270 BPM. Then the program branches to the validate and update routines. The validate routine first checks if

$$
V_{F 3}>T F
$$

and

$$
1.5 V_{F 3}>V_{F 1}
$$

where TF is the threshold used to detect $V_{F 1}$. These conditions mean that the fetal $R$ peak was obtained in a very noisy signal. Otherwise, similar checks are made with $\mathrm{V}_{\mathrm{F} 2}$, where

$$
V_{F 2}>T F
$$

and

$$
1.5 V_{F 2}>V_{F 1}
$$

also imply a noisy signal.

If $\mathrm{V}_{\mathrm{F} 1}$ is the only maximum above the threshold then it is taken as a fetal $R$ wave. If $V_{F 2}$ also exceeds the threshold, then $V_{F 1}$ is checked for coincidence with possible spikes by relating its instant to the four maternal values which are kept in the record. The spike position, $t_{s}$ and the position, $t_{F 1}$ in the signal associated with the local maximum, are compared for

$$
t_{S}-t_{F 1}<40 m s
$$

which allows for the difference in correlation delay when obtaining $t_{S}$ and $t_{F 1}$ respectively. If $V_{F 1}$ is identified as a large spike in the signal, then $V_{F 2}$ and $V_{F 3}$ are assumed to be the fetal $R$ peak and the noise, respectively. 
Thresholds and search interval limits are updated according to the procedure described earlier and the local maxima values are then set to zero for the subsequent $\mathrm{R}$ wave search.

\subsection{System realization in hardware}

The QRS detection algorithm was initially implemented in Visual $\mathrm{C}++$ because it is simpler and faster to verify the functionality and reliability. Then, the algorithm was implemented in VHDL; where Altera's Quartus II version 4.0 is used as the platform. As a result, for VHDL implementation the algorithm has to be thought of as a structural, behavioral and physical version of the algorithm. The advantage of using Quartus $I I$ is that the system can be synthesized into a physically available FPGA, or the built-in simulation device models. The built-in simulation device models emulate the real device with the actual timing and power values. Thus, its performance in terms of timing, speed, power consumption, total logic element counts and functionality can be ascertained. Modifications can be easily performed and its impact to the physical implementation, especially in terms of timing and functionality can be immediately known.

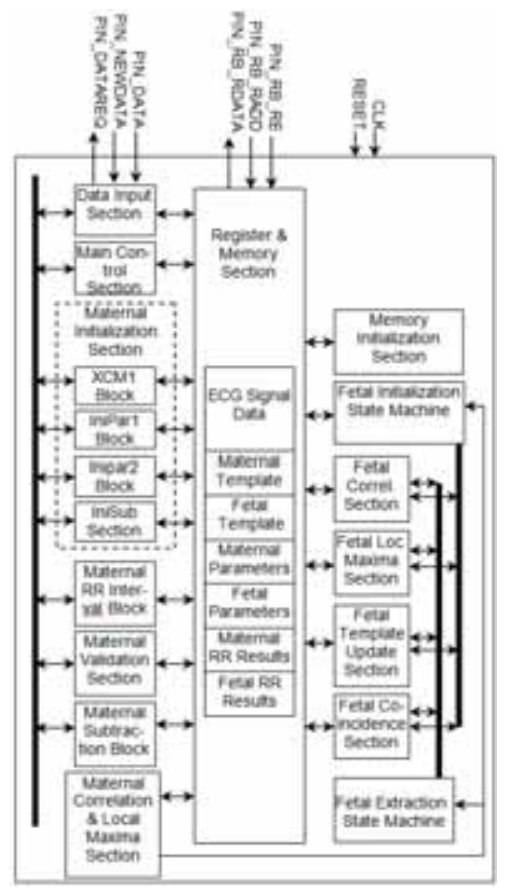

Figure 2. Top level block diagram.
Figure 2 shows a simplified block diagram of the implementation of the system. Basically, the system is categorized into three main sections, the common, maternal and fetal sections. The common block, shared by both maternal and fetal sections are the data input section, memory register section, memory initialization section and main control section. Maternal blocks consist of the maternal initialization sections (XCM1 block, inipar1 block, maternal inipar2 block and iniSub block), maternal RR interval block, maternal validation block, maternal subtraction block and maternal correlation \& local maxima section. Fetal blocks consist of fetal initialization section, fetal extraction section, fetal correlation section, fetal local maxima search section, fetal template update section and fetal coincidence section. The pins for the system PIN_NEWDATA, PIN_DATA and PIN_DATAREQ are used to interface with an external module to retrieve new data. When the system is done, PIN_RB_RE, PIN_RB_RADD and PIN_RB_RDATA are used to access the DPRAM to retrieve the stored maternal and fetal RR interval results from their corresponding memory segment.

\section{Results and discussion}

\subsection{Data structure}

In this research, the signals used were downloaded from MIT database website. The signals consist of both normal AECG and AECG with paroxysmal atrial fibrillation. The test signals consist of both normal and atrial fibrillation ECG.

The data set consists of 50 record sets. Each record set contains two 30-minute with consecutive record names (e.g., n01 and n02), and two 5minute "continuation" records with names ending in c (e.g., n01c and n02c). All four records in each record set are excerpts of longer continuous AECG recordings of a single subject. The 50 record sets come from 48 different subjects. The records with names beginning with $\mathrm{p}$ come from subjects who have paroxysmal atrial fibrillation (PAF). The second (even-numbered) record in each pair of 30minute records contains the ECG immediately preceding an episode of PAF. Thus, for example, record p16 immediately precedes the episode of the PAF in record p16c. 
The first (odd-numbered) record of the set (for example, record p15) contains 30 minutes of the AECG during a period that is distant from any episode of PAF. Distant means there is no PAF during the 45-minute period before or after the 30minute record in this case. The corresponding 5minute continuation record (e.g., record p15c) shows the minutes immediately following the PAFdistant record.

The records with names beginning with $n$ come from subjects who do not have documented atrial fibrillation, either during the period from which the records were excerpted or at any other time. The subjects include healthy controls, patients referred for long-term ambulatory ECG monitoring, and patients in intensive care units.

All signals are converted into text format and graphical format in excel files.

The *.dat files downloaded are in binary format. They contain the digitized ECGs with 16 bits per sample, least significant byte first in each pair, 128 samples per signal per second, samples from each channel alternating, nominally 200 A/D units per millivolt.

They are manually converted to text file format *.txt by using a C++ program, confil.cpp. Since the total number of samples is large (128 samples per second $=7680$ samples per minute, thus total samples $=7680 * 40=307200$ samples per subject), only two thousand samples are converted in each text file $(2000 \star 4=8000$ samples per subject).

\subsection{Visual C++ and VHDL simulation}

The result in table 1 using Visual $\mathrm{C}++$ shows encouraging results with the test case, where the fetal $\mathrm{R}$ peak could be detected up to $98 \%$. Upon completion of the test case simulation using VHDL, the system performs a read request to retrieve all the maternal and fetal results. A sample read operation for maternal and fetal $\mathrm{R}$ peaks detection is shown in figure 3 with the initial 4900 sample data. The Quartus simulation result shows that the
VHDL models are functioning almost similar to the Visual $\mathrm{C}++$ function. The results for both versions are shown in table 2 and 3. Comparing the maternal and fetal RR interval values (in terms of number of samples between the intervals), the maternal error is consistently less than $0.3 \%$, and the fetal error percentage is within $0.5 \%$.

All the differences are caused by the rounding effect during computation. However, when a fetal peak loss happens, an error rate up to $4 \%$ might have occurred, owing to slightly different search limit implemented in the VHDL. Despite this, the VHDL interpretation of the system displays great similarities to the Visual $\mathrm{C}++$ version.

\begin{tabular}{|l|l|l|}
\hline \multicolumn{2}{|l|}{ Description } & Value \\
\hline Maternal & Total R peak & 164 \\
\hline & Detected & $164(100 \%)$ \\
\hline Fetal & Total R peak & 235 \\
\hline & $\begin{array}{l}\text { Detected using first } \\
\text { threshold }\end{array}$ & $182(77 \%)$ \\
\cline { 2 - 3 } & $\begin{array}{l}\text { Detected using } \\
\text { second threshold }\end{array}$ & $5(02 \%)$ \\
\cline { 2 - 3 } & Coincidence & $46(19 \%)$ \\
\hline
\end{tabular}

Table 1. Test simulation using Visual $\mathrm{C}++$.

\begin{tabular}{|l|l|l|l|}
\hline \multirow{2}{*}{ Sample No. } & \multicolumn{2}{|l|}{ RR intervals } & \multirow{2}{*}{$\%$ Diff } \\
\cline { 2 - 3 } & VC++ & VHDL & \\
\hline 1 & 297 & 297 & $0 \%$ \\
\hline 2 & 299 & 299 & $0 \%$ \\
\hline 3 & 301 & 301 & $0 \%$ \\
\hline 4 & 295 & 295 & $0 \%$ \\
\hline 5 & 296 & 297 & $0.3 \%$ \\
\hline 6 & 294 & 293 & $-0.3 \%$ \\
\hline 7 & 297 & 297 & $0 \%$ \\
\hline 8 & 292 & 292 & $0 \%$ \\
\hline 9 & 299 & 298 & $-0.3 \%$ \\
\hline 10 & 296 & 295 & $-0.3 \%$ \\
\hline 11 & 296 & 295 & $-0.3 \%$ \\
\hline 12 & 290 & 291 & $0.3 \%$ \\
\hline 13 & 289 & 288 & $-0.3 \%$ \\
\hline 14 & 290 & 291 & $0.3 \%$ \\
\hline
\end{tabular}

Table 2. Comparison between VHDL and Visual $\mathrm{C}++$ results detecting number of maternal RR intervals. 


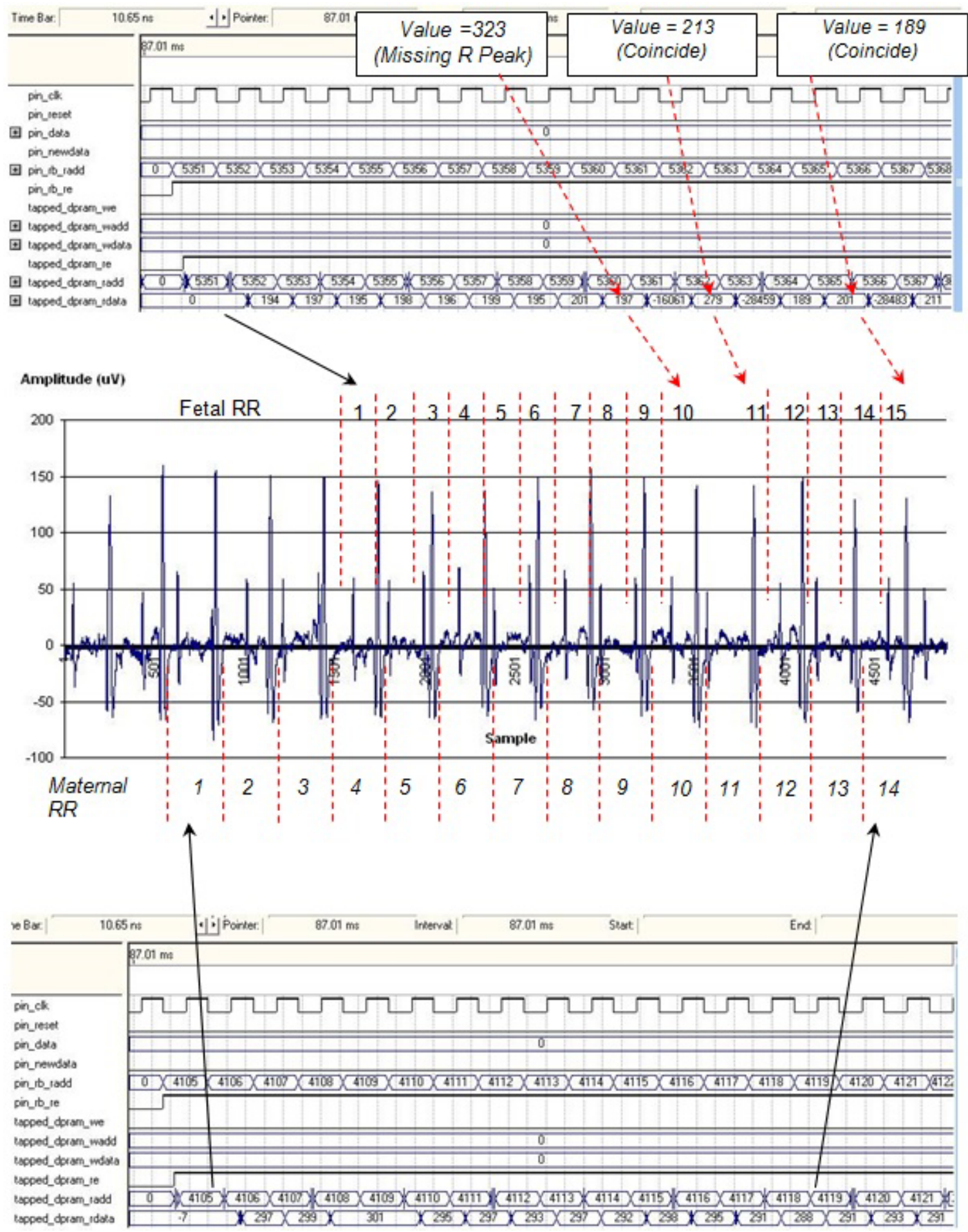

Figure 3. R peak detection of maternal and fetal with 4900 data. 


\begin{tabular}{|c|c|c|c|}
\hline \multirow{2}{*}{ Sample No. } & \multicolumn{2}{|c|}{ RR Intervals } & \multirow{2}{*}{$\%$ Diff. } \\
\cline { 2 - 3 } & VC++ & VHDL & \\
\hline 1 & 194 & 194 & $0 \%$ \\
\hline 2 & 197 & 197 & $0 \%$ \\
\hline 3 & 195 & 195 & $0 \%$ \\
\hline 4 & 197 & 198 & $0.5 \%$ \\
\hline 5 & 197 & 196 & $-0.5 \%$ \\
\hline 6 & 198 & 199 & $0.5 \%$ \\
\hline 7 & 196 & 195 & $-0.5 \%$ \\
\hline 8 & 201 & 201 & $0 \%$ \\
\hline 9 & 197 & 197 & $0 \%$ \\
\hline 10 & 312 & 323 & $3.5 \%$ \\
\hline 11 & 290 & 279 & $-3.8 \%$ \\
\hline 12 & 212 & 213 & $0.5 \%$ \\
\hline 13 & 190 & 189 & $-0.5 \%$ \\
\hline 14 & 201 & 201 & $0 \%$ \\
\hline
\end{tabular}

Table 3. Comparison between VHDL and Visual C++ Results detecting number of fetal RR intervals.

\subsection{Synthesis}

Table 4 and 5 show the resources consumed during compilation to fit the system into an Altera's Stratix device. The total resources required are calculated, allocated and then fitted into Altera Stratix EP1S10 device. The tables below show the number of logic elements, registers, memory and DSP units used during fitting and compilation. The DSP units are used for the implementation of the accumulator and multiplication between the signal and template points for both the fetal and maternal correlation block.

\begin{tabular}{|l|l|}
\hline Description & Total \\
\hline Device & EP1S10F780C5 \\
\hline Netlist size & 10376 Nodes \\
\hline Usage & $9,633 / 10,570(91 \%)$ \\
\hline Total memory bits & $101,616 / 920,448(11 \%)$ \\
\hline DSP blocks & $4 / 48(08 \%)$ \\
\hline Total pins & $346 / 427(81 \%)$ \\
\hline
\end{tabular}

Table 4. Summary of Resource Fitting.

\begin{tabular}{|c|c|c|c|c|c|}
\hline \multicolumn{2}{|l|}{ Block } & Logic cells & Register & Memory bits & DSP \\
\hline \multicolumn{2}{|l|}{ Top } & 9,633 & 1586 & 101,616 & 4 \\
\hline \multirow{3}{*}{ Common } & Data input block & 33 & 30 & 0 & 0 \\
\hline & Initialization block & 143 & 55 & 0 & 0 \\
\hline & Registers and memory block & 1,489 & 742 & 101,616 & 0 \\
\hline \multirow{16}{*}{ Maternal } & Initialization and extraction block & 1,053 & 130 & 0 & 0 \\
\hline & a. Main state machine & 198 & 36 & 0 & 0 \\
\hline & b. Inipar1 sub-block & 174 & 48 & 0 & 0 \\
\hline & c. Inipar2 sub-block & 254 & 0 & 0 & 0 \\
\hline & d. Initxcm1 state machine & 191 & 10 & 0 & 0 \\
\hline & e. Inisubtract state machine & 236 & 36 & 0 & 0 \\
\hline & Correlation block & 516 & 107 & 0 & 2 \\
\hline & RR Interval block & 315 & 6 & 0 & 0 \\
\hline & Validation block & 1,224 & 151 & 0 & 0 \\
\hline & a. Main state machine & 936 & 91 & 0 & 0 \\
\hline & b. Error check state machine & 288 & 60 & 0 & 0 \\
\hline & UpMatPar block & 730 & 0 & 0 & 0 \\
\hline & Subtraction lock & 502 & 114 & 0 & 0 \\
\hline & a. Main state machine & 156 & 48 & 0 & 0 \\
\hline & b. MPK state machine & 258 & 56 & 0 & 0 \\
\hline & c. XCM2 state machine & 88 & 10 & 0 & 0 \\
\hline \multirow{7}{*}{ Fetal } & Initialization block & 1,003 & 15 & 0 & 0 \\
\hline & a. Main state machine & 414 & 15 & 0 & 0 \\
\hline & b. Coincidence sub-block & 589 & 0 & 0 & 0 \\
\hline & Extraction block & 1630 & 35 & 0 & 0 \\
\hline & Correlation block & 290 & 100 & 0 & 2 \\
\hline & Local Maximum block & 233 & 42 & 0 & 0 \\
\hline & Template block & 472 & 59 & 0 & 0 \\
\hline
\end{tabular}

Table 5. Fitter resource allocation. 


\section{Conclusion}

The performance achieved for the heart rate measurements from the AECG shows that the model can extract both R-peak of maternal and fetal utilizing a single-lead configuration. The result using Visual $\mathrm{C}++$ shows that the fetal $\mathrm{R}$ peak could be detected up to $98 \%$. Test case results also showed an error percentage of around $\pm 0.3 \%$ and $\pm 0.5 \%$ for the R-peak detection of maternal and fetal respectively. The system is capable of running at a maximum clock frequency of 48.56MHz, and consumed 9633 logic elements, 101616 memory space and 4 units of DSP blocks. Therefore, we can concluded that the developed algorithm and hardware approach will be a useful tool in the assessment of the fetal condition and its relationship to that of the mother's.

\section{References}

[1] R. K. Freeman et al., "Fetal heart rate monitoring" Philadelphia, USA, Lippincott Williams \& Wilkins, 2003.

[2] J. Jezewski et al., "Comparison of Doppler ultrasound and direct electrocardiography acquisition techniques for quantification of fetal heart rate variability", IEEE Transactions on Biomedical Engineering, vol. 53, no. 5, pp. $855-864,2006$.

[3] M. M. S. Algunaidi et al., "Evaluation of an improved algorithm for fetal QRS detection", International Journal of the Physical Sciences, vol. 6, no. 2, pp. 213-220, 2011.

[4] M. A. Hasan et al., "Detection and processing techniques of FECG signal for fetal monitoring", Biological Procedures online, vol. 11, no. 1, pp. 263-295, 2009.

[5] M. Ungureanu et al., "Improved method for fetal heart rate monitoring", 27th Annual International Conference of the Engineering in Medicine and Biology Society, Shanghai, China, 2005, pp 5916- 5919.

[6] T. Fukushima et al., "Limitations of autocorrelation in fetal heart rate monitoring", American Journal Obstetrics and Gynecology. vol. 153, no. 6, pp. 685-692, 1985.

[7] H. Helgason et al., "Adaptive multiscale complexity analysis of fetal heart rate", IEEE Trans Biomed Eng, vol. 58, no. 8, pp. $2186-2193,2011$.
[8] T. Solum et al., "The accuracy of abdominal ECG for fetal electronic monitoring", Journal of prenatal medicine, vol. 8 no. 3, pp. 142-149, 1980.

[9] P. Maria et al., "Monitoring the fetal heart noninvasively: A review of methods", Journal of Prenatal Medicine, vol. 29, no. 5, pp. 408-416, 2001.

[10] P. Coussy et al., "An introduction to high-level synthesis", IEEE Design \& Test of Computers, vol. 26, no. 4, pp. 8-17, 2009.

[11] M. B. I. Reaz et al., "Prototyping of wavelet transform, artificial neural network and fuzzy logic for power quality disturbance classifier, Journal of Electric Power Components and Systems", vol. 35, no. 1, pp. 117, 2007

[12] M. Akter et al., "Hardware implementations of image compressor for mobile communications", Journal of Communications Technology and Electronics, vol. 53, no. 8, pp. 899-910, 2008.

[13] M. B. I. Reaz et al., "FPGA realization of multipurpose FIR filter", The Parallel and Distributed Computing, Applications and Technologies (PDCAT), Chengdu, China, 2003, pp. 912-915.

[14] F. Mohd-Yasin et al., "The FPGA prototyping of Iris recognition for biometric identification employing neural network", The International Conference on Microelectronics, Tunis, Tunisia, 2004, pp. 458-461.

[15] M. B. I. Reaz and L. S. Wei, "Adaptive Linear Neural Network Filter for Fetal ECG Extraction", The International Conference on Intelligent Sensing and Information Processing, Chennai, India, 2004, pp. 321324.

[16] S. Abboud et al., "Quantification of the fetal Electrocardiogram using averaging technique", Computers in Biology and Medicine, vol. 20, no. 3, pp. 147-155, 1990.

[17] J. Pan and W. J. Tompkins, "A real-time QRS detection algorithm", IEEE Transactions on Biomedical Engineering, vol. 32, no. 3, pp. 230-235, 1985.

[18] S. Azevedo and R. L. Longini, "Abdominal-lead fetal Electrocardiographic R-Wave enhancement for heart rate determination", IEEE Transactions on Biomedical Engineering, vol. 27, no. 1, pp. 255-260, 1980. 